\title{
Estructura y textura de un meteorito metálico de tipo octaedrita (Gibeon)
}

\author{
J. GIL SEVILLANO, C. GARCíA-ROSALES, JON J. ECHEBERRIA Y C. ZUBILLAGA \\ Centro de Estudios e Investigaciones Técnicas de Guipúzcoa (CEIT) y Escuela Superior de Ingenieros Industriales \\ (Universidad de Navarra), Paseo de Manuel Lardizábal, 15, 20009 San Sebastián
}

\begin{abstract}
Se ha estudiado un fragmento del hierro meteorítico Gibeon (Namibia, 1847), con estructura de octaedrita fina, combinando técnicas de microscopía óptica, microscopía electrónica de barrido, análisis EDX, medida de orientaciones cristalográficas mediante EBSP (Electron Back Scattered Patterns) y difracción de rayos X. La muestra (aprox. 24x23x3 mm, $14.3 \mathrm{~g}$ ) presenta la clásica estructura de Widmanstätten, producto de la intersección de placas lenticulares de ferrita (kamacita), orientadas según planos octaédricos, con la sección metalográfica de la muestra. Se observan también una fracción residual de austenita (taenita) retenida entre las placas ferríticas y estructuras más complejas (plesita), producto de la descomposición extraterrestre de la última fracción de austenita. La muestra presenta pocas evidencias de modificaciones por impacto (bandas de Neumann, etc.), pero sí abundantes subgranos en la kamacita, separados por juntas de débil desorientación $\left(<2^{\circ}\right)$. La macrotextura obtenida a partir de figuras de polos (difracción de rayos X) y las medidas individuales de orientaciones (EBSP) evidencian la relación aproximada de orientaciones austenita/ferrita de Kurdjumov-Sachs (24 variantes) y el plano de hábito $\{111\}_{\gamma} /\{110\}_{\alpha}$ de las placas de ferrita, así como el carácter monocristalino de la austenita original de este fragmento (aunque la dispersión de orientaciones de la austenita tampoco es despreciable).
\end{abstract}

Palabras clave: meteorito, octaedrita, estructura Widmanstätten, relación de orientaciones, Kurdjumov-Sachs, textura.

\section{Strcuture and crystallographic texture of a metallic meteorite of octahedrite type (Gibeon)}

A sample from the meteoritic iron Gibeon (fine octahedrite) has been studied combining optical and scanning electron microscopy, crystallographic microtexture by EBSP (Electron Back Scattered Patterns) and macrotexture by X-ray diffraction. The sample (approx. $24 \times 23 \times 3 \mathrm{~mm}, 14.3 \mathrm{~g}$ ) shows the classical Widmastätten structure of intersecting ferrite (kamacite) plates grown along $\{111\}_{\gamma}$ octahedric previous austenite (taenite) planes. Residual layers of austenite are also seen between the ferrite plates as well as larger domains of more complex structures of bainitic type (plessite) produced by the last fraction of austenite transformed during extra-terrestrial cooling. The sample structure shows few signs of impact modifications (Neumann bands, etc.). Kamacite plates are divided in weakly misoriented $\left(<2^{\circ}\right)$ subgranular domains. The measurement of individual orientations by EBSP or the macroscopic texture measurement show a single austenite orientation (although with a non-negligible dispersion around the average orientation) and a limited number of ferrite orientations compatible with the variants of the Kurdjumov-Sachs orientation relationship.

Keywords: meteorite, octahedrite, Widmanstätten structure, orientation relationship, Kurdjumov-Sachs, texture.

\section{INTRODUCCIÓN}

Además de por su valor geológico-cosmológico como piezas minerales de origen extraterrestre, testigos quizá de los procesos de formación de los cuerpos celestes, los meteoritos metálicos, básicamente aleaciones Fe-Ni, constituyen muestras de muy alto interés desde el punto de vista de la Ciencia e Ingeniería de Materiales, porque han sufrido transformaciones de fase desde el estado líquido a velocidades de enfriamiento absolutamente fuera del alcance de cualquier experimento de laboratorio, del orden de $10^{-7}$ a $10^{-5}{ }^{\circ} \mathrm{C} /$ año (1-5). Sus estructuras, por tanto, representan el resultado de condiciones presuntamente próximas a las ideales de transformación en equilibrio termodinámico. Esto es especialmente interesante para comparar las estructuras de transformación en fase sólida con las estructuras de aleaciones similares obtenidas por transformación isotérmica o por enfriamiento continuo a velocidades accesibles a la experimentación y la técnica. En concreto, se observa en los meteoritos metálicos una solidificación monocristalina o un tamaño de grano enorme (decímetros o metros, maclas de decímetros de espesor, por ejemplo) de la fase soli- dificada (austenita -taenita en la terminología meteorítica- para la mayoría de los meteorítos metálicos). La ausencia o baja densidad de juntas de grano hace que la transformación posterior en fase sólida (de austenita a ferrita - kamacita) sea mayoritariamente intragranular y conduzca también a tamaños de grano inalcanzables en muestras de laboratorio.

Existe en este momento un fuerte interés técnico en el desarrollo de estructuras de ferrita nucleada intragranularmente para conseguir aceros de alta resistencia y alta tenacidad (la denominada "ferrita acicular"), cuya morfología y cristalografía son asombrosamente coincidentes con la de las octaedritas meteoríticas, aunque la escala dimensional es unas $10^{3}$ veces menor y la velocidad de enfriamiento con la que se consiguen, unas $10^{4}$ ó $10^{5}$ veces mayor (6-11). El estudio de las estructuras Widmanstätten meteoríticas y la comprensión de su proceso de formación (nucleación y crecimiento) puede ofrecer claves muy interesantes para una mejor comprensión de la producción de estructuras de ferrita acicular en los aceros (y viceversa). 


\section{PROCEDIMIENTO EXPERIMENTAL}

\subsection{Muestra estudiada.}

La muestra estudiada, de $14.3 \mathrm{~g}$ y dimensiones aproximadas $24 \times 23 \times 3 \mathrm{~mm}$, está cortada de un fragmento proveniente de Gibeon (Namibia, caída múltiple, descubierto en 1838, registrados $21000 \mathrm{Kg}$ (3)). La muestra fue adquirida en 1998 en el Museo de Mineralogía de L'École des Mines de Paris (exposición organizada por ABC Mines) y su superficie había sufrido un lijado fino y un fuerte ataque que permitía ver claramente la típica estructura Widmanstätten de los hierros meteóricos. Para el estudio que se describe en este trabajo, la muestra fue preparada mediante un lijado y pulido metalográficos convencionales, con un acabado usando sílice coloidal (Struers OP-U, 0,04 $\mu \mathrm{m}$ ). Se hicieron medidas de texturas por difracción de rayos $X$ y observaciones de microscopía óptica y electrónica de barrido (SEM) de la muestra pulida sin atacar, aunque la austenita quedaba levemente realzada por su mayor dureza. Posteriormente, la muestra se atacó ligeramente con Nital al 2\% (fig. 1) para continuar el examen metalográfico.

Los ejemplares del meteorito Gibeon provienen de un cuerpo de gran tamaño que probablemente se fragmentó a su paso por la atmósfera. Se han recogido ( y se siguen recogiendo en la actualidad) un gran número de fragmentos originales cuya masa oscila entre $195 \mathrm{~g}$ y $650 \mathrm{Kg}$. Es uno de los meteoritos metálicos más limpios de inclusiones no metálicas y pertenece al grupo IV A Of. (7.5 a 9.5\% en peso de níquel, octaedrita fina, esto es, espesor de las placas de kamacita de 0.2 a $0.5 \mathrm{~mm})$.

\subsection{Técnicas de observación y análisis.}

La superficie de la muestra atacada se ha observado por microscopía óptica (a pocos aumentos, en campo claro; detalles, usando contraste interferencial). Mediante MEB (Philips XL-30), la imagen de electrones retrodispersados permite distinguir suficientemente bien las distintas fases y las subestructuras de la muestra simplemente pulida. Las orientaciones individuales de ambas fases presentes se han determinado en distintas áreas de la muestra mediante análisis de patrones de Kikuchi (EBSP, electron back-scattered patterns) con un sistema automatizado TSL (TexSEM Laboratories, Inc.). El sistema TSL permite también obtener imágenes asociadas a las orientaciones cristalinas del área analizada o a las desorientaciones presentes. Se han realizado también algunos análisis locales de composición a partir del espectro de energías de rayos $X$ emitido en el MEB (EDX, equipo EDAX). La macrotextura de la ferrita y austenita de la zona central de aprox. 10×10 mm de la muestra se ha medido también por difracción de rayos X. Este método no es el más adecuado para una muestra de estas características (austenita monocristalina y grano muy grueso de la ferrita respecto a materiales técnicos) y las figuras de polos obtenidas no son de gran precisión. Sin embargo, el análisis combinado de 4 figuras de polos por fase ha permitido calcular una FDO (función de distribución de orientaciones) consistente y en buen acuerdo con las medidas de microtextura por EBSP. Se ha utilizado un goniómetro de texturas Philips X'Pert, radiación $\mathrm{k}_{\alpha}$ del Co, software Philips PC-Texture de toma de datos y análisis de figuras de polos, y software MTS-FHM (12) de cálculo de FDO.

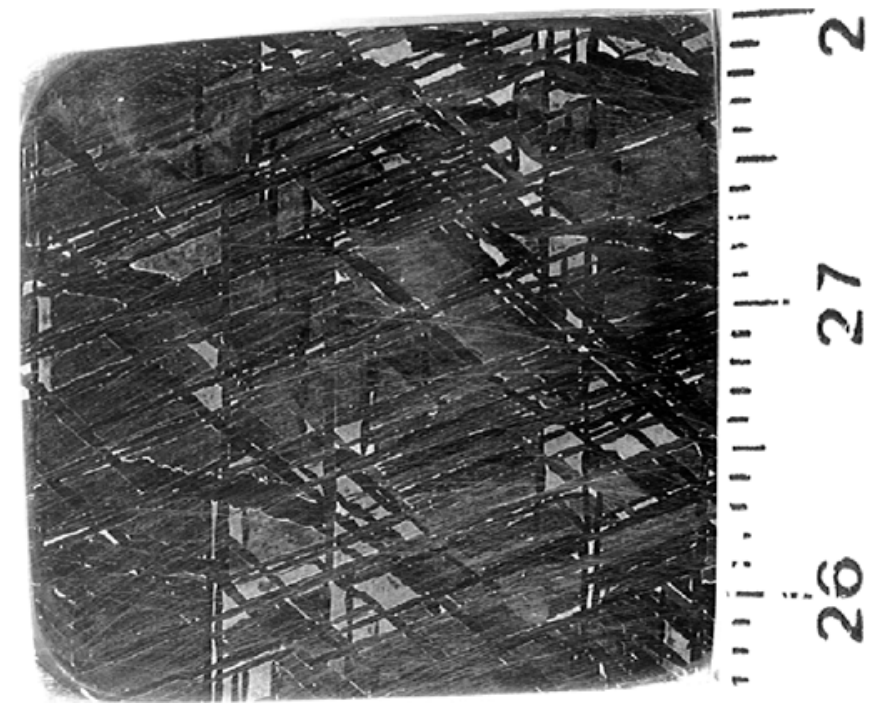

Figura 1. Macrografía de la muestra completa (atacada con Nital). La normal al lado inferior se tomó como dirección 1 del sistema de referencia usado para la medida de texturas por rayos $\mathrm{X}$ y para las fotografías de microscopía óptica (la dirección 3 es la normal al plano de la imagen).

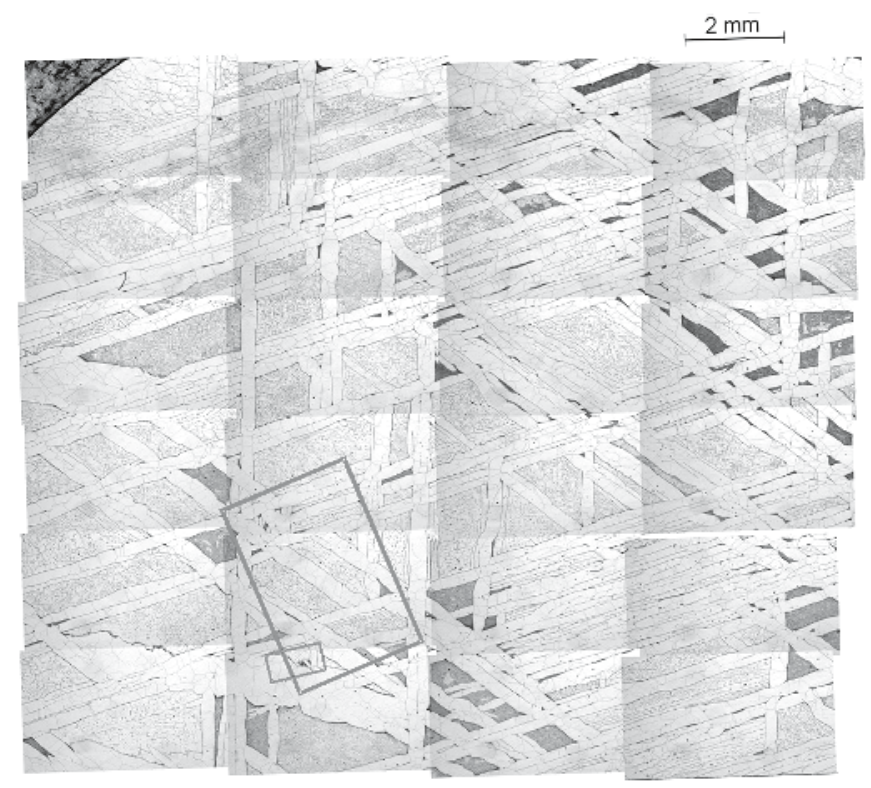

Figura 2. Composición de micrografías ópticas (campo claro) de la muestra atacada con Nital.

\section{RESULTADOS.}

\subsection{Microscopía óptica.}

La figura 2 es una composición de 24 fotografías tomadas con el microscopio óptico. La orientación es la misma de la figura 1 y el área ampliada puede reconocerse en la zona central izquierda. Se observan 4 orientaciones espaciales de placas de ferrita (placas blancas), limitadas en muchos casos por capas muy delgadas de austenita (negro) y, entre las placas, amplias zonas triangulares o trapezoidales subestructuradas de distintos tipos de "plesita". La estructura, fracción superficial de fases y sus tamaños coinciden indudablemente con las imágenes publicadas de otras muestras de Gibeon (véase, por ejemplo, la entrada correspondiente a Gibeon en el "Handbook 


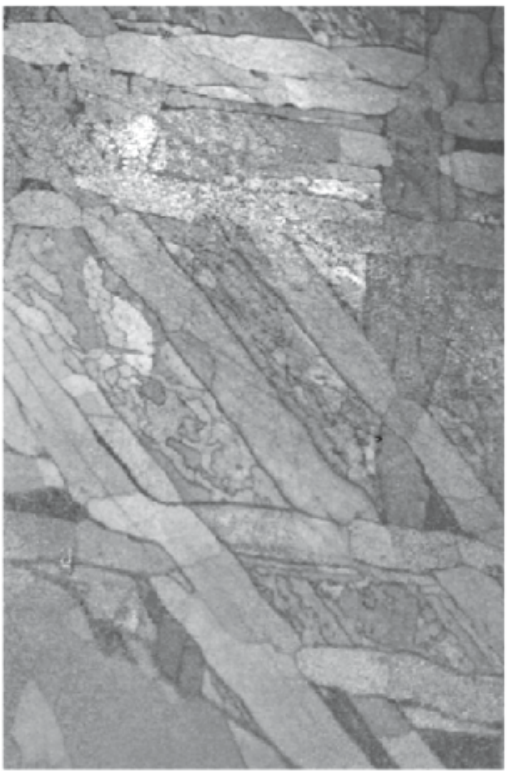

$900.0 \mu \mathrm{m}=60 \mathrm{steps}$

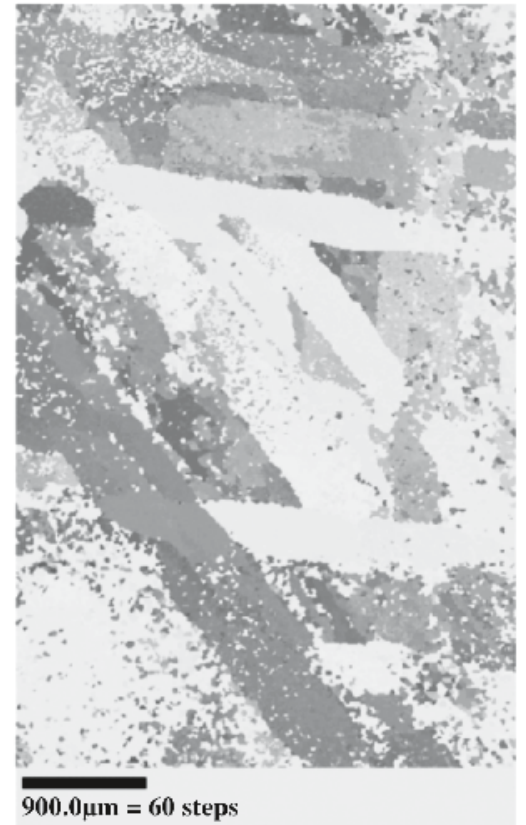

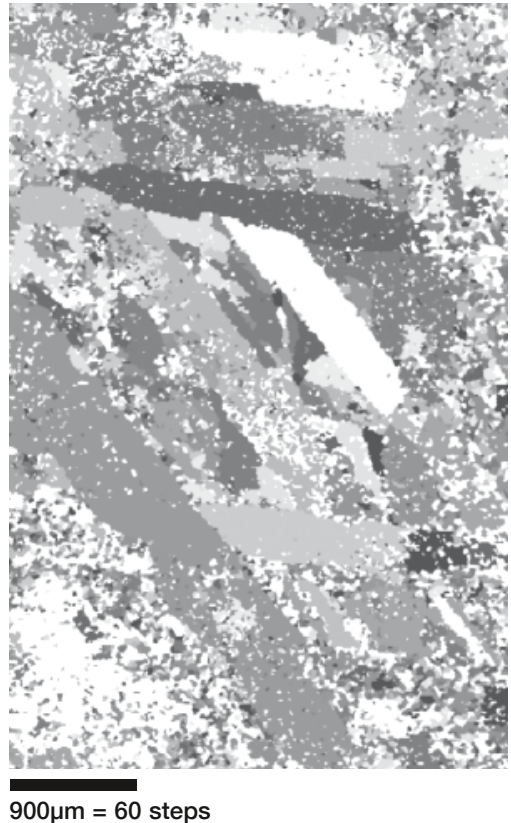

$900 \mu \mathrm{m}=60$ steps

Figura3. a) Imagen basada en el "índice de calidad del patrón de Kikuchi" de la zona encuadrada de la fig. 1, obtenida a partir de determinaciones de orientación cada $15 \mathrm{~mm}$. Las zonas más distorsionadas aparecen más oscuras; b) Id., imagen de color codificada según la dirección cristalográfica normal al plano de la figura en cada punto (imagen de la figura de polos inversa); c) Id., imagen de dominios desorientados más de $2^{\circ}$.

of Metallic Meteorites", (13)). Gibeon es conocido por su policristalinidad (granos originales de austenita de 100 a $500 \mathrm{~mm}$, muchos de ellos con maclas de espesor entre 50 y $100 \mathrm{~mm}$ ), pero no se detecta en la muestra ningún signo de junta original de austenita. La estructura, al menos a esta escala, no manifiesta modificaciones de impacto ni distorsiones artificiales. $\mathrm{Ni}$ siquiera se ven bandas de Neumann claras, frecuentes en Gibeon. Incluso a estos aumentos se distinguen subgranos en la placas de ferrita, limitados por subjuntas frecuentemente paralelas o transversales a la traza del plano de hábito de las placas. El espesor de la sección de las placas más delgadas coincide con el espesor real citado para la kamacita de este meteorito $(0.30 \pm 0.05 \mathrm{~mm}$ (13)). A estos aumentos y en esta sección, apenas se detectan inclusiones $\mathrm{u}$ otras fases distintas de ferrita y austenita; al otro lado de la muestra se ve a simple vista una típica inclusión de troilita, $\mathrm{SFe}$, de unos $2 \times 3 \mathrm{~mm}$. Se ha detectado también algún silicato de forma aproximadamente esférica.

\subsection{Microscopía electrónica de barrido (MEB y OIM).}

La zona encuadrada de la fig. 2 se muestra en la fig. 3. La imagen 3a es un mapa basado en el "índice de calidad del patrón de Kikuchi" (14), relacionado con la distorsión local de la red cristalina. Pone en evidencia zonas deformadas, subjuntas y fronteras de grano. La austenita residual aparece como una línea negra que delimita en muchos puntos las placas de ferrita. Entre las láminas de ferrita aparecen regiones de plesita celular subdivididas en dominios de unas $100 \mu \mathrm{m}$. Otras regiones más oscuras corresponden a variedades de plesita de más baja temperatura de transformación (son resultado de las últimas transformaciones bainíticas o martensíticas de la austenita no transformada en placas de kamacita de primera generación [3]). Los detalles estructurales se distinguen mejor en las imágenes MEB de electrones retrodispersados, por ejemplo, figs. 4 y 5 . Existe toda una jerarquía de estructuras.

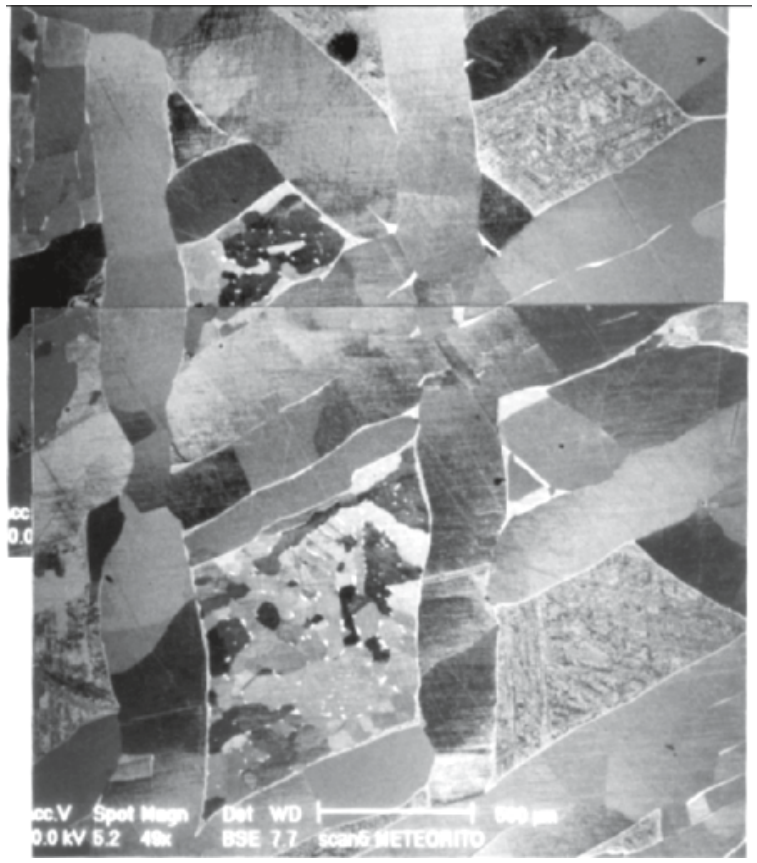

Figura 4. Imagen MEB de electrones retrodispesados de una zona próxima a las anteriores. Muestra no atacada. La austenita residual, con mayor contenido en níquel que la ferrita, se ve más clara. La zona rectangular de plesita celular parece una zona transformada en placas de ferrita (kamacita) de $2^{a}$ generación, con restos de austenita formando islas. La zona trapezoidal adyacente tiene rasgos bainí-

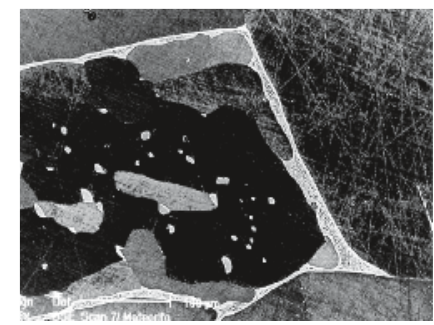

Fig.5 Detalle de la figura 4 ticos o martensíticos. Las trazas de las placas son las mismas en todos los casos. 
La figura $3 b$ es un mapa de polos cristalinos normales al plano de la imagen 3a (codificación de color según la figura inversa de polos anexa). Pueden distinguirse variantes distintas que comparten la misma orientación octaédrica de la austenita original, es decir, placas de ferrita con el mismo plano de hábito, indistinguibles salvo por su orientación cristalina. La resolución de la figura es demasiado grosera para detectar detalles y muchos puntos aparecen blancos por no alcanzar un índice aceptable de seguridad en el cálculo de la orientación (además de puntos individuales debidos a imperfecciones superficiales de la muestra y que se han de interpretar como ruido). La figura 3 c es un mapa que distingue desorientaciones superiores a $2^{\circ}$. En la mayoría de los casos, los subgranos de las placas primarias de ferrita, cuyas subjuntas se ven muy bien en las imágenes ópticas, están muy débilmente desorientados.

En la fig. 6, a más aumentos, es posible distinguir bien la austenita de la ferrita. En la fig.7 se presentan tres figuras de polos de cada fase; la austenita es claramente monocristalina (las orientaciones dispersas son probablemente errores de medida por defectos superficiales) y las orientaciones de la ferrita corresponden aproximadamente a las 24 variantes derivadas de la relación de Kurdjumov-Sachs a partir de la orientación de la austenita original:

(111) $\gamma / /(110) \alpha$

$[1-10]_{\gamma} / /[1-11]_{\alpha}$

Claramente, pueden descartarse las relaciones, más simples, de Bain o de Nishiyama-Wassermann. Un ejemplo exacto de relación K-S se muestra, para comparación, en la fig. 7.

\subsection{Textura obtenida por difracción de rayos $\mathrm{X}$.}

La medida de texturas de monocristales o multicristales mediante difracción de rayos $\mathrm{X}$ es poco apropiada y la técnica mejor a emplear con un meteorito metálico para determinar con buena precisión su macrotextura sería la difracción de neutrones, que puede proporcionar información de un gran volumen. Sólo conocemos un caso en que se haya medido la textura de un meteorito metálico, precisamente por difracción de neutrones, pero se trata de un meteorito mal documentado y el análisis que se hizo fue muy somero (5). Las figuras de polos directas que se obtuvieron de Gibeon fueron de poca calidad, al estar enormemente concentrados los picos de difracción, especialmente en la austenita. Sin embargo, combinando la información de 4 figuras de polos por fase y obteniendo la FDO compatible con las 4 figuras de polos, se ha obtenido un resultado muy aceptable y consistente con una única orientación cristalina de austenita y una textura de transformación de ferrita concordante aproximadamente con K-S, fig. 8. La orientación de las figuras de polos es coincidente con la de las figs. 1 y 2 . Puede comprobarse que las 4 trazas de los planos $\{111\}_{\mathrm{g}}$ concuerdan con las orientaciones espaciales de las intersecciones de las placas de ferrita con el plano de la muestra, figs. 1 y 2.

\section{DISCUSIÓN}

Es la primera vez que se presenta un análisis tan detallado de las orientaciones cristalográficas locales asociadas a la microestructura de un meteorito metálico, junto con un análisis de la macrotextura de la misma muestra. Sin embargo, se trata sólo de un estudio preliminar que deja entrever las posibilidades actuales de combinación de técnicas de observación disponibles para el estudio de los materiales. Algunas cuestiones que surgen de este estudio y que se pretende desarrollar en trabajos posteriores se refieren a la formación de las placas

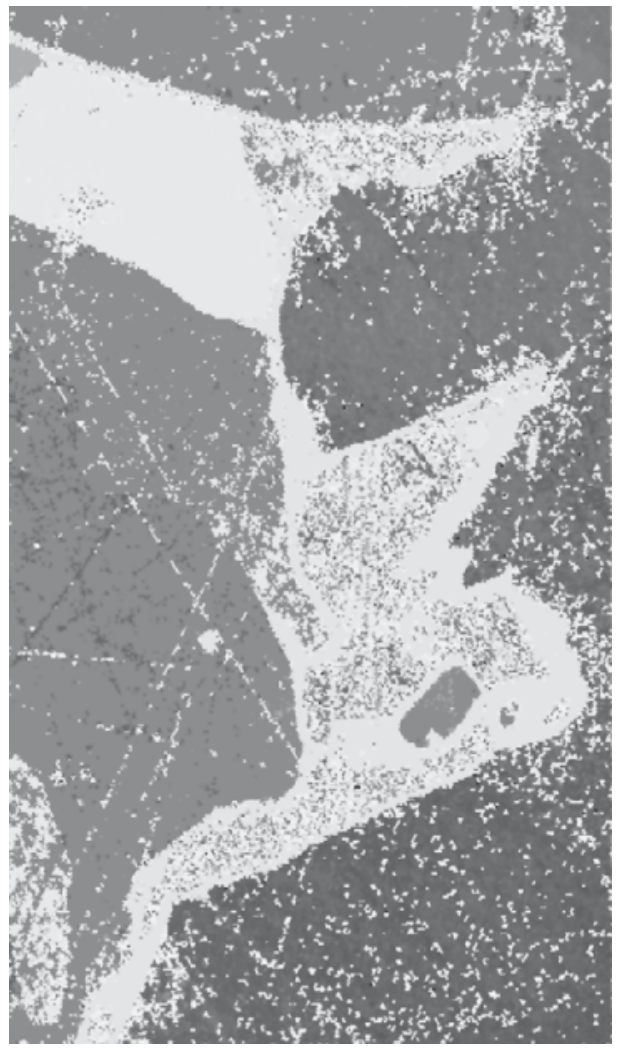

Figura 6. Detalle de la zona inferior izquierda de la fig. 3. OIM, idéntica codificación de colores que para la fig. $3 \mathrm{~b}$. La zona verde es austenita residual monocristalina, aprox. normal a $\langle 110\rangle$; separa áreas de ferrita diferentemente orientadas y encierra una zona bifásica muy finamente subestructurada.

Widmanstätten de ferrita en los meteoritos. La bibliografía acepta generalmente que son producto de una transformación totalmente por difusión, dados los tiempos de enfriamiento del cuerpo original del que proviene el meteorito. Al mismo tiempo, las dificultades de nucleación intragranular evidenciadas por el enorme tamaño de las placas de ferrita y el gradiente de composición de las capas de austenita residual o transformada a baja temperatura sugieren un subenfriamiento importante respecto a la temperatura de equilibrio del diagrama de fases Fe-Ni $\left(50-100^{\circ} \mathrm{C}\right.$ (3)). Para la composición de Gibeon, se estaría cerca o por debajo de $\mathrm{T}_{0}$, la temperatura de equilibrio de austenita y ferrita de la misma composición[16-17], temperatura límite para que la transformación no sea totalmente difusional. Algunos detalles de las imágenes mostradas en este trabajo podrían sugerir que ha existido cierta interacción en el crecimiento de dos placas de ferrita, por ejemplo en la parte central derecha de la fig. 3, como si la placa vertical hubiera sido deformada a cortadura por la placa inclinada, en la zona de intersección de las dos placas. Hay un desplazamiento de la placa vertical a ambos lados de la intersección y un pequeño cambio de orientación cristalográfica del volumen presuntamente afectado. Habría que verificar si tales interacciones se repiten consistentemente en la muestra. También, mediante OIM es interesante comprobar si se da repetidamente algún tipo de emparejamiento preferente de placas que comparten el mismo plano de hábito pero corresponden a variantes diferentes; tal emparejamiento contribuiría a facilitar la nucleación y crecimiento de las placas por mecanismos no reconstructivos. 

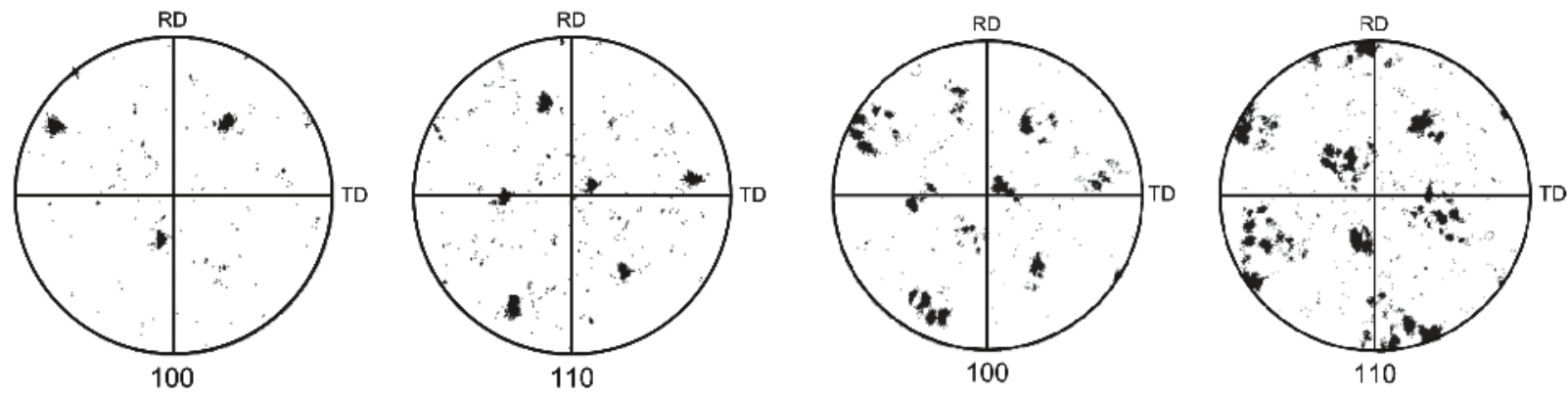

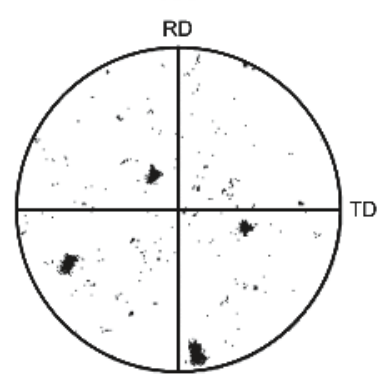

FCC

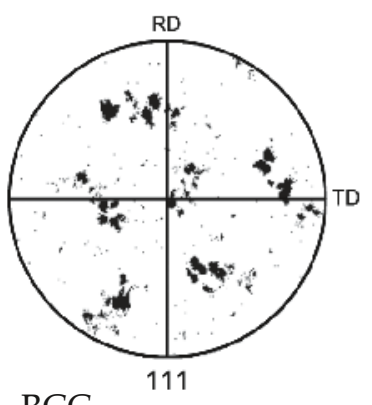

BCC

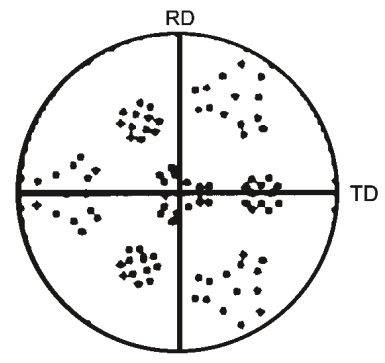

Figura 7. b)Figura de polos $<110>\alpha$ de las 24 variantes K-S transformadas de la orientación (111)[110] $\gamma$. Reproducido de (15).

Figura 7. a) Figuras de polos de la austenita (FCC) y de la ferrita (BCC) correspondientes a las orientaciones individuales medidas para obtener la figura 6 anterior.

Meteorito: Austenita.
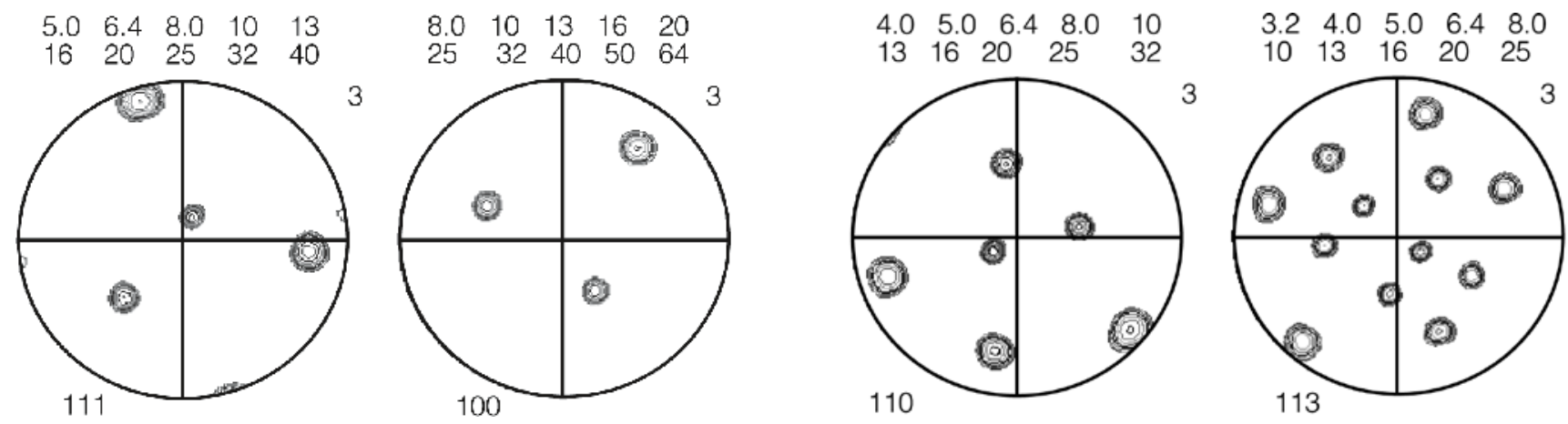

Meteorito: Ferrita.
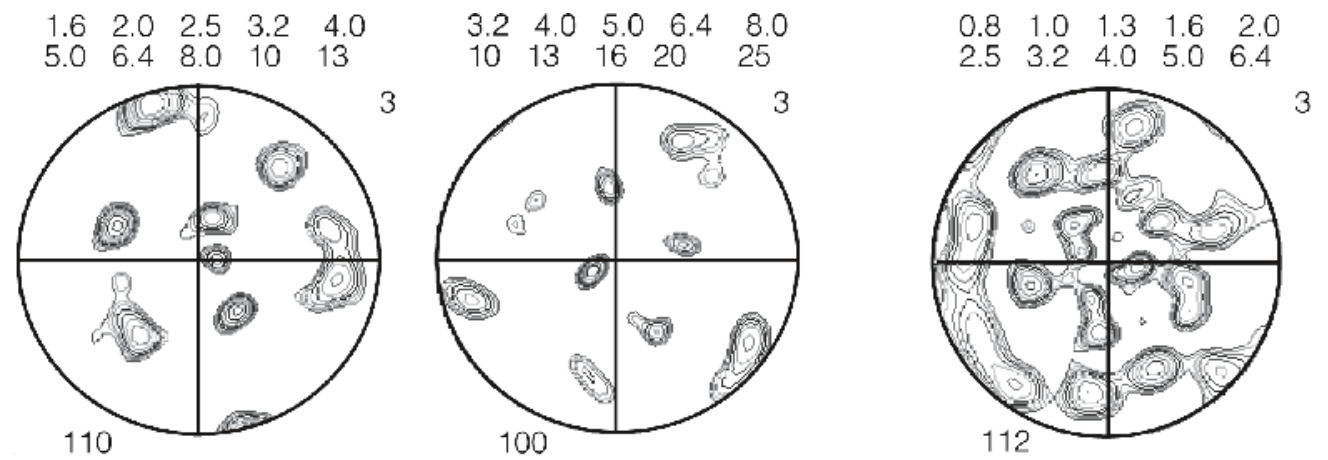

$\begin{array}{lllll}1.3 & 1.6 & 2.0 & 2.5 & 3.2\end{array}$ $\begin{array}{lllll}4.0 & 5.0 & 6.4 & 8.0 & 10\end{array}$

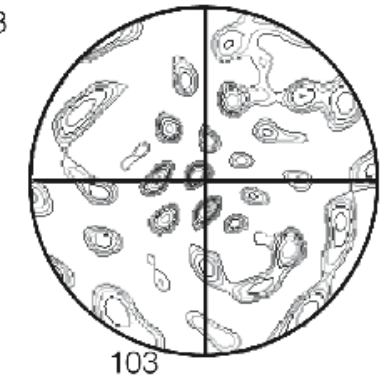




\section{AGRADECIMIENTOS}

J. G. S. agradece sinceramente a Javier García Guinea y Jesús Martínez Frías, Departamento de Geología del CSIC, la entusiasta acogida en su despacho del Museo de Ciencias Naturales de Madrid y el haber hecho posible la consulta de bibliografía de otra manera inalcanzable.

\section{BIBLIOGRAFÍA}

1. Heide, F. y Wlotzka, F., "Meteorites. Messengers from Space". SpringerVerlag, Berlín, 1995.

2. Zanda, B. y Rotaru, eds., "Les Météorites", Muséum National d'Histoire Naturelle, París. Bordas, París, 1996.

3. Buchwald, V. F., "Handbook of Iron Meteorites", 3 vols., Univ. of California Press, Berkeley, USA, 1975.

4. Axon, H. J., "Metallurgy of Meteorites", Progr, Mater. Sci., 13, 183 (1968).

5. Helmig, K. Nikitin, A. N. and Walther, "Neutron texture investigation of a meteor", Text. Microstr., 14-18, 279-282 (1991).

6. Bhadeshia, H. K. D. K., "Shear transformations in steels", Acta Mater., 29, 1117-1130 (1981)

7. Ali, A. y Bhadeshia, H. K. D. K., " Microstructure of high-strength steel refined with intragranularly nucleated Widmanstátten ferrite", Mater. Sci. Technol., 7, 895-903 (1991).
8. Honeycombe, R. W. K. y Bhadeshia, H. K. D. H., "Steels. Microstructure and Properties", 2a ed., Edward Arnold, London, 1995.

9. Madariaga, I., "Ferrita acicular en aceros microaleados de contenido medio en carbono: nucleación, microestructura y comportamiento mecánico". Tesis de Doctorado, Facultad de Ciencias, Universidad de Navarra, 1999.

10. Madariaga, I., Romero, J. L. y Gutiérrez, I., "Upper acicular formation in a medium-carbon microalloyed steel by isothermal transformation: nucleation enhancement by CuS". Metall. And Mater. Trans., vol. 29A, 1003 (1998).

11. Madariaga, I. y Gutiérrez, I., "Role of the particle-matrix interface on the nucleation of acicular ferrite in a medium carbon microalloyed steel". Acta Mater., vol. 47, 951 (1999).

12. Van Houtte, P., "The MTM-FHM software system Version 2", Katholieke Universiteit Leuven, Bélgica.

13. Id., ref. 3, vol. 2, p. 584

14. "TSL - CEIT Academy on EBSD and OIM and their Applications" (notas del curso). San Sebastián, 20 al 30 de junio, 1999.

15. Yasuda, H. Y., Sakata, T. y Umakoshi, Y, "Variant selection in transformation texture from the $\mathrm{b}$ to a phase in $\mathrm{Cu}-40$ mass \% $\mathrm{Zn}$ alloys", Acta Mater., vol. 47, 1923 (1999).

16. Chuang, Y. Y., Hsieh, K. C. y Chang, Y. A., “A thermodynamic analysis of the phase equilibria of the Fe-Ni system above $1200 \mathrm{~K}$ ", Metall. Trans. A, vol. 17A, 1373 (1986)

17. Chuang, Y. Y., Chang, Y. A., Schmid, R. y Lin, J. C., “Magnetic contribution to the thermodynamic functions of alloys and the phase equilibria of $\mathrm{Fe}-\mathrm{Ni}$ system below 1200 K", METALL. TRANS. A, vol. 17A, 1361 (1986). 